\title{
Reprint: Peculiarities of expression of apoptosis markers in the tissues of primary fallopian tubes carcinoma
}

Franklin Unawunwa*, Natalia Hyriavenko, Anna Korobchanska, Mykola Lyndin, Vladyslav Sikora

Sumy State University, Belgium.

*Corresponding Author: Franklin Unawunwa, Sumy State University, Belgium.

Received Date: September 01, 2021 | Accepted Date: October 09, 2021 | Published Date: October 14, 2021

Citation: F Unawunwa, N Hyriavenko, A Korobchanska, M Lyndin, V Sikora. (2021). Peculiarities of expression of apoptosis markers in the tissues of primary fallopian tubes carcinoma. Biomedical Research and Clinical Reviews. 5(1); DOI:10.31579/2692-9406/084

Copyright: () 2021 Franklin Unawunwa, This is an open-access article distributed under the terms of the Creative Commons Attribution License, which permits unrestricted use, distribution, and reproduction in any medium, provided the original author and source are credited.

Aim: immunohistochemical analysis of apoptosis markers in the tissue of PFTC.

Introduction: Primary fallopian tubes carcinoma is a rare case among oncological diseases of female genital organs, but the mortality rate is rather high. Nowadays, the prognostic factors of this neoplasia are not fully determined. The data on the p53 and bcl 2 proteins expression and their use as prognostic factors in patients with malignant tumors of many locations are contradictory.

Methods: the study was conducted on 66 samples of fallopian tubes tumor tissue. To study the apoptosis peculiarities of tumor cells the mouse monoclonal antibodies for bcl-2 (clone 100/D5) and p53 (clone SP5) were used. Mathematic calculations were done using Microsoft Excel 2010 with AtteStat 12.0.5.

Results: The high expression of p53 was found in patients of all clinical stages. Mutations of p53 increased with spreading of the neoplastic process. Strong correlation of p53 presence in tumor samples and clinical stage of the disease was determined ( $r=0.77)$. In contrast to the abovementioned protein the study of bcl-2 showed the moderate negative correlation between this protein and the stage of the disease $(r=-0.54)$. Analysis of the dependence of p53 expression with the presence or absence of lymph nodes metastasis showed a direct correlation between the indicators ( $r=0.25$ ). Thus the level of p53 expression in patients with N1 was 80.6 $\pm 2.7 \%$ compared with the N0 group (29.7 $\pm 3.6 \%)$. The stage of neoplasia differentiation is in moderate direct correlation with p53 expression $(r=0.58)$ and in inverse with - bcl-2 $(r=-0.64)$.

Conclusion: Expression of p53 depends on neoplasia spreading and stage of tumor differentiation. The expression of p53 is an independent prognostic marker for $\mathrm{N}$-status and helps to classify the patients into "risk" groups.

Acknowledgements: Supervisor: A. M. Romaniuk, prof., doctor of medical sciences, Department of Pathological Anatomy, Medical Institute, Sumy State University. 